\title{
On moral hazard and nonexclusive contracts
}

\author{
Andrea Attar $^{1,2}$ and Arnold Chassagnon ${ }^{3,4}$
}

\begin{abstract}
We study an economy where intermediaries compete over contracts in a nonexclusive insurance market affected by moral hazard. In this context, we show that, contrarily to what is commonly believed, market equilibria may fail to be efficient even if the planner is not allowed to enforce exclusivity of trades (third best inefficiency). Our setting is the same as that of Bisin and Guaitoli (2004). We hence argue that some of the equilibrium conditions they imposed are not necessary, and we exhibit a set of equilibrium allocations which fail to satisfy them.
\end{abstract}

Keywords: Non-exclusivity, Insurance, Moral Hazard.

JEL Classification: D43, D82, G22.

This work analyzes nonexclusive financial markets where intermediaries strategically compete in the presence of moral hazard on the agents' side. Non-exclusivity refers to a scenario where every agent (consumer-borrower) can simultaneously accept the proposals of several intermediaries, and her decisions cannot be contracted upon.

Many researches have tried to provide a theoretical assessment of these situations. The study of non-exclusive relationships under moral hazard has been initiated by Pauly (1974), Hellwig (1983) and Arnott and Stiglitz (1993), and first reformulated by Bizer and DeMarzo (1992) and Kahn and Mookherjee (1998). These last contributions, though, examined the particular setting where agents take their contractual decisions sequentially. More recently, Parlour and Rajan (2001), Martimort and Stole (2003), Attar, Campioni, and Piaser (2006) and others have focused on the standard model of competition where intermediaries simultaneously post their contract offers and, in a next step, agents take their portfolio and effort decisions. A general analysis of this framework is proposed in Bisin and Guaitoli (2004), who study a standard insurance setting without any restriction on players' preferences. ${ }^{1}$ Overall, these researches emphasize that whenever exclusive trades cannot be enforced, positive profits for the active intermediaries and a distorted distribution of surplus typically arise. A crucial condition to support this sort of non-competitive outcomes in an otherwise competitive set-up is the presence of latent contracts. These are offers that are not bought at equilibrium, which role is to guarantee a threat against the incentive of a single intermediary to increase his market share by reducing his price.

\footnotetext{
${ }^{1}$ Toulouse School of Economics (IDEI-GREMAQ).

${ }^{2}$ Università di Roma II, Tor Vergata.

${ }^{3}$ Toulouse School of Economics (GREMAQ).

${ }^{4}$ PSE, Ecole Normale Supérieure, Paris.
}

We thank Alberto Bisin and an anonymous referee for their extremely useful comments on a previous draft of this work. We are also grateful to G. Bloise, E. Campioni, M. Hellwig, G.Piaser, U. Rajan and to seminar participants at Universitat de Rosario, at the JMA meeting (Nantes), the 2006 SED conference (Bodrum), and the 2006 EGRIE conference (Barcelona). All responsibility remains to the authors.

${ }^{1}$ Their analysis can be extended to consider credit relationships without any loss of generality (see Section 4 and 5 of Bisin and Guaitoli (2004)). 
A major issue in this literature is the analysis of the welfare effects of non-exclusivity. In markets where trades are nonexclusive, a single intermediary cannot write contracts which are contingent on agents' portfolio decisions, which induces a form of contractual externalities.

The relevant welfare results can be resumed as follows: if contractual externalities are sufficiently severe, then market equilibria fail to be constrained (second best) efficient. A social planner who cannot control agents' effort choices may indeed perform better than markets as long as he retains the power of controlling trades. In a next step, one can consider the situation where the planner cannot restrict financial intermediaries from offering further trade opportunities. This seems a natural scenario to investigate in all those situations where a social planner is in the impossibility of enforcing exclusivity clauses. The related efficiency notion is referred to as third best optimality. All recent works in this line (see Kahn and Mookherjee (1998), Bisin and Guaitoli (2004) and Attar, Campioni, and Piaser (2006)) have shown an efficiency result: equilibria are incentive-constrained inefficient, but third-best efficient. $^{2}$

The first contribution of the present work is to provide a simple example of a set of equilibrium allocations in a nonexclusive insurance market subject to moral hazard which fail to be third best efficient. If the planner is facing the same informational constraints as the intermediaries, there exist feasible allocations which are preferred to the equilibrium ones by both intermediaries and agents. We hence show that even in such a constrained scenario there is room for regulatory issues: public insurance provision turns out to be welfare improving.

As a matter of fact, our analysis also delivers new insights at a positive level. Indeed, we consider the same general framework of Bisin and Guaitoli (2004) (BG) who introduce a set of necessary conditions for pure strategy equilibria. In addition, they emphasize the role of latent contracts to support high effort at equilibrium. We characterize a set of equilibrium allocations which are supported by only one active intermediary, who earns a positive profit. The amount of latent insurance needed at equilibrium is higher than what conjectured by BG (and its corresponding price is lower). Interestingly, these situations give rise to to an insufficient provision of insurance from the only active intermediary. In the light of our results, we hence argue that some of the properties identified by BG are not necessarily verified at equilibrium. This is the second contribution of our work.

The paper is organized as follows: section 1 presents the model and provides our example, section 2 develops the welfare analysis, and section 3 provides a discussion of our results. All proofs are collected in the appendix.

\section{Equilibria under non-exclusivity: an example}

We consider an insurance economy lasting two periods. It is populated by a single representative consumer and by a countably infinite set of intermediaries. ${ }^{3}$ The probability distribution over the set of idiosyncratic states $\{1,2\}$ depends on an unobservable effort $e=\{a, b\}$. Uncertainty affects the consumer's endowment $w=\left(w_{1}, w_{2}\right) \in \mathbb{R}_{+}^{2}$, with $w_{2}>w_{1}: \pi_{a}\left(\pi_{b}\right)$ is taken to be the probability of occurrence of state 2 if $a(b)$ is chosen, with $a>b$ and $\pi_{a}>\pi_{b}$. Trades are represented by insurance contracts offered by intermediaries to the single consumer. Every intermediary $i \in J$ offers

\footnotetext{
${ }^{2}$ If contractual externalities are sufficiently mild, then market equilibria can be efficient in the sense of second best even in the presence of rationing. This is the case, for instance, in Bizer and DeMarzo (1992) and Parlour and Rajan (2001) (see Attar, Campioni, and Piaser (2006) for a discussion).

${ }^{3}$ Alternatively, one can think of a continuum of identical consumers.
} 
a contract $d^{i}=\left(d_{1}^{i}, d_{2}^{i}\right)$ consisting in a pair of state-contingent transfers and we take $p_{i}=\left|\frac{d_{2}^{i}}{d_{1}^{i}}\right|$ to be the price of the $d^{i}$ contract. The agent could buy a fraction $\lambda_{i}$ of this contract. In addition, every $i-t h$ intermediary fixes $\Lambda_{i}$ (the set of admissible $\lambda_{i}$ ) to be either $[0,1]$ (divisible contracts) or $\{0,1\}$ (indivisible contracts). ${ }^{4}$ Insurance relationships are nonexclusive: the consumer chooses a subset of intermediaries to trade with and her decisions cannot be contracted upon.

The payoff to intermediary $i$ is given by $V^{i}=-\left(\pi_{e} d_{2}^{i}+\left(1-\pi_{e}\right) d_{1}^{i}\right) \lambda_{i}$, when the effort $e$ is chosen and the fraction $\lambda_{i}$ of the offer $d^{i}$ is bought. We denote $\vec{\pi}_{e}=\left(\left(1-\pi_{e}\right), \pi_{e}\right)$, and we then write $V^{i}=$ $-\vec{\pi}_{e} \cdot d^{i} \lambda_{i}$. The agent-consumer is risk-averse. Her utility from consuming $c_{s}$, with $s=\{1,2\}$, is given by $u: \mathbb{R}_{+} \rightarrow \mathbb{R}_{+}$, which is continuous, increasing and concave. The corresponding expected utility is $\tilde{U}\left(c_{1}, c_{2}, e\right)=\left(1-\pi_{e}\right) u\left(c_{1}\right)+\pi_{e} u\left(c_{2}\right)-e$, where $e$ denotes the cost of effort and $c_{s}=w_{s}+\sum_{i \in J} \lambda_{i} d^{i}$ is the contingent consumption. In what follows, we will always refer to $U(C)=\tilde{U}(C, e(C))$, with $e(C) \in \underset{e}{\arg \max } \tilde{U}(C, e)$. We also take $\mathcal{A}=\left\{C \in \mathbb{R}_{+}^{2} / e(C)=a\right\}$ to be the set of ex-post consumption profiles inducing the choice $e=a$ and $\mathcal{B}=\left\{C \in \mathbb{R}_{+}^{2} / e(C)=b\right\}$ to be that inducing $e=b$.

We will henceforth take the consumer's utility to be $u(c)=c^{\gamma}$, with $\gamma \in(0,1)$. Consider the following players' behaviors:

i) $d^{1}=C-W, \quad \Lambda^{1}=\{0,1\}$,

ii) $d^{2}=d^{3}=L-C, \quad \Lambda^{2}=\Lambda^{3}=[0,1]$,

iii) $d^{4}=d^{5}=\cdots=d^{J}=(0,0)$,

iv) $\lambda_{1}=1, \lambda_{2}=\lambda_{2}=\cdots=\lambda_{J}=0$, and $e=a$, with $C=\left(c_{1}, c_{2}\right) \in \mathcal{A}, L=\left(l_{1}, l_{2}\right) \in \mathcal{B}, c_{1}>w_{1}$, and $c_{2}<w_{2}$. That is, intermediary 1 makes a positive insurance, take-it or leave-it offer (indivisible contract), while intermediaries 2 and 3 propose a divisible contract, and all other intermediaries offer the null contract $(0,0)$. The consumer accepts the offer of intermediary 1 and rejects those of intermediaries 2 and 3, as well as the remaining ones. In addition, she selects the high effort.

Figure 1 depicts the consumer's feasible consumption set given the endowment $W$ and the offers' array $\left(d^{1}, d^{2}, \cdots, d^{J}\right)$.

\footnotetext{
${ }^{4}$ The case of indivisibility clearly denotes a situation where the $i-t h$ intermediary is making a take-it or leave-it offer.
} 


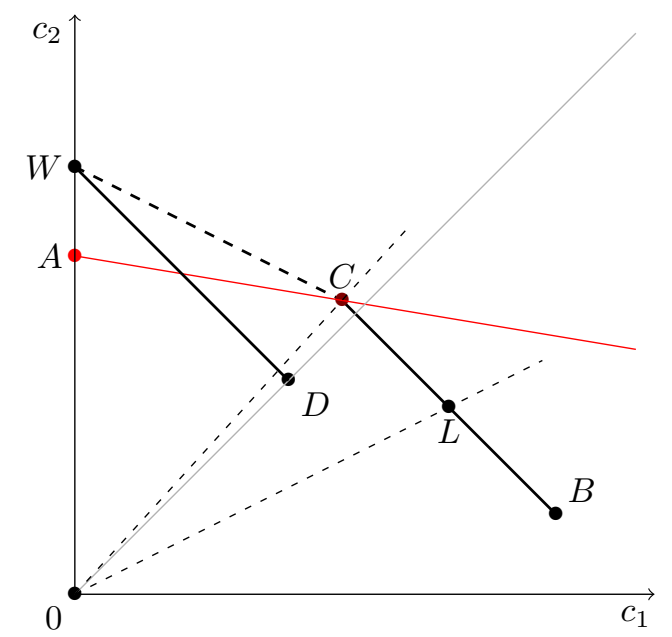

Figure 1: Set of feasible consumption allocations

The array $(C, L)$ is identified by the following conditions:

$$
\begin{gathered}
U(C)=U(L) \\
\tau^{L} c_{1}+c_{2}=\tau^{L} l_{1}+l_{2} \\
\pi_{a}\left(1-\pi_{a}\right)\left(u^{\prime}\left(c_{1}\right)-u^{\prime}\left(c_{2}\right)\right)=\pi_{a}\left(1-\pi_{b}\right) u^{\prime}\left(l_{1}\right)-\pi_{b}\left(1-\pi_{a}\right) u^{\prime}\left(l_{2}\right) \\
\tau^{b}>\tau^{L}>\tau^{C}>\left|\frac{w_{2}-c_{2}}{w_{1}-c_{1}}\right|>\tau_{a}, \\
w_{1}+2\left(l_{1}-c_{1}\right)=w_{2}+2\left(l_{2}-c_{2}\right), \\
\frac{w_{2}+3\left(l_{2}-c_{2}\right)}{w_{1}+3\left(l_{1}-c_{1}\right)}<\frac{l_{2}}{l_{1}}, \quad-\vec{\pi}_{a} \cdot(L-C)<-\vec{\pi}_{a} \cdot(C-W), \\
u\left(\frac{p_{1}-\tau^{a}}{\tau^{C}-\tau^{a}}\right) \leq \frac{1}{\gamma} u^{\prime}\left(\frac{c_{2}}{\tau^{L} c_{1}+c_{2}} \frac{\tau^{L}-\tau^{a}}{\tau^{C}-\tau^{a}} \frac{1}{\pi_{a}}\right)
\end{gathered}
$$

where $\tau^{L}=\frac{\left(1-\pi_{b}\right) u^{\prime}\left(l_{1}\right)}{\pi_{b} u^{\prime}\left(l_{2}\right)}$ and $\tau^{C}=\frac{\left(1-\pi_{a}\right) u^{\prime}\left(c_{1}\right)}{\pi_{a} u^{\prime}\left(c_{2}\right)}$ denote the marginal rate of substitution of the consumer evaluated at $L$ and at $C$, respectively. In addition, we denote $\tau^{a}=\frac{1-\pi_{a}}{\pi_{a}}$ and $\tau^{b}=\frac{1-\pi_{b}}{\pi_{b}}$. Relationships (1)-(7) have a straightforward interpretation: (1) requires the single agent to be indifferent between $C$ and $L$, and (2) states that the price of the contracts offered by intermediaries 2 and 3 is $\tau^{L}$. Considered together, (1), (2) and (3) identify the role of the latent allocation $L$ as a threat to prevent unilateral deviations to positive insurance contracts. The choice of $e=b$ will indeed make these deviations not profitable, since it raises the price effectively paid to the consumer. Suppose that intermediary $i$ deviates to $d^{i \prime}=d^{i}+d$, where $d$ is such that $-\vec{\pi}_{a} \cdot d^{i \prime}>-\vec{\pi}_{a} \cdot d^{i}$. The deviation is profitable if $e=a$ is chosen. Condition (3) implies that following any of such deviations, the consumer will have an incentive to accept it together with the allocation $L$ (provided it is available) and to select $e=b$ as the optimal effort choice. Since $d$ can be very small, it follows that (1) will also hold. Furthermore, (2) states that the threat $L$ remains available if any of the intermediaries removes his offer. There should hence be enough latent insurance issued at equilibrium, which implies that the slope of the line connecting $C$ and $L$ will be equal to $\tau^{L}$. The inequalities in (4) provide additional elements 
for equilibrium characterization: the first one guarantees that $L$ will fall in the overinsurance region, at the right of the 45 degree. The second and the third one state that accepting the offer $d^{1}=C-W$ is an optimal choice for the consumer when she selects $e=a$. The last inequality implies that the only active intermediary earns a positive profit offering $d^{1}$, at a price $p_{1}=\left|\frac{w_{2}-c_{2}}{w_{1}-c_{1}}\right|$.

Conditions (5) and (6) imply that the threat of selecting the low effort will still be effective even when large deviations are considered. More precisely, (5) states that whenever the active intermediary removes his offer, there will be enough available insurance to get to the 45 degree line. The inequalities in (6) further characterize the amount of latent insurance available at equilibrium. In particular, the last inequality puts an upper bound on the supply of inactive intermediaries: its role is to prevent deviations through negative insurance contracts.

Finally, condition (7) introduces a limit to the price charged by the active intermediary. As will be clarified in the following, if the inequality is violated there might be gains from price undercutting. Summarizing: given $W$ and the offers' array $\left(d^{1}, d^{2}, \ldots, d^{J}\right)$, the agent can achieve the allocation $C$ as well as every element on the line connecting $C$ and $L$ with slope $-\tau^{L}$ (see Figure 1).

The main contribution of the present work is therefore to establish the following:

Proposition 1 Consider an economy where consumer's preferences are represented by $u(c)=c^{\gamma}$, with $\gamma \in(0,1)$. Then, for every array $(C, L)$ satisfying $(1)-(7)$, the allocation $C \in \mathcal{A}$ can be supported as a pure strategy equilibrium by the players' behaviors described in $(i)-(i v)$.

The proposition is proved in three Lemmas. The first one shows that the set of allocations $(C, L)$ satisfying $(1)-(7)$ is non-empty. The second stresses the restrictions induced by these conditions on the consumer's optimal behavior. Finally, lemma 3 argues that the described players' behaviors form an equilibrium. As a starting point, we therefore establish:

Lemma 1 If $u(c)=c^{\gamma}$, with $\gamma \in(0,1)$, there exists an array of parameters $\left(W, \gamma, \pi_{a}, \pi_{b}, a, b\right)$ such that the system (1) - (7) has a solution $(C, L)$, with $C \in \mathcal{A}$ and $L \in \mathcal{B}$.

Importantly, one can show the existence of a continuum of points in the neighborhood of $(C, L)$ that can be as well supported at a pure strategy equilibrium. ${ }^{5}$ This guarantees the robustness of the result. We now consider the consumer's behavior, emphasizing the features of her optimal choices which are relevant to analyze the deviation stage. To this extent, we refer to the allocation $C_{\alpha} \in \mathcal{A}$. For every $\alpha \in \mathbb{R}, C_{\alpha}$ is taken to be at the intersection between the (iso-profit) line of slope $-\frac{1-\pi_{a}}{\pi_{a}}$ passing through $C$ and the line connecting $\alpha C$ and $\alpha L$ : every $C_{\alpha}$ hence guarantees the same aggregate profit as that earned by the single intermediary in in $C$. That is: $C_{\alpha}=\left(c_{1}+\pi_{a} f(\alpha), c_{2}-\left(1-\pi_{a}\right) f(\alpha)\right)$, with $f^{\prime}(\alpha)>0$ and $f(1)=0 .^{6}$

Lemma 2 If $u(c)=c^{\gamma}$, with $\gamma \in(0,1)$, and the allocations $C$ and L satisfy $(1)-(7)$, then:

1. $U\left(C_{\alpha}\right)<U(\alpha L) \quad \forall \alpha \neq 1$

2. $U(K)<U(\alpha L) \quad \forall K \neq C$ lying between $C_{\alpha}$ and $\alpha L$,

3. $U(K)<U(\beta L) \quad \forall K, \forall \beta \geq 1$ such that $p_{1} k_{1}+k_{2} \leq p_{1} c_{\beta 1}+c_{\beta 2}$ and $\tau^{L} k_{1}+k_{2} \leq \tau^{L} c_{\beta 1}+c_{\beta 2}$.

\footnotetext{
${ }^{5}$ The detailed computation is available from the authors.

${ }^{6}$ The reader can check that $f(\alpha)=\frac{\alpha-1}{\pi_{a}} \frac{\tau^{L} c_{1}+c_{2}}{\tau^{L}-\tau^{a}}$ and that $\tau^{L} c_{1}+c_{2}=\pi_{a} f^{\prime}(\alpha)\left(\tau^{L}-\tau^{a}\right)$.
} 
The lemma stresses the main properties of the consumer's optimal choices. In particular, the first two inequalities show that the consumer will always prefer to select the low effort $e=b$ instead of taking $e=a$ and choosing an allocation providing an aggregate profits to the intermediaries greater than $-\vec{\pi}_{a} \cdot C$. The last one identifies the set of allocations that are dominated from the consumer point of view by $\beta L$ (see Figure 2).

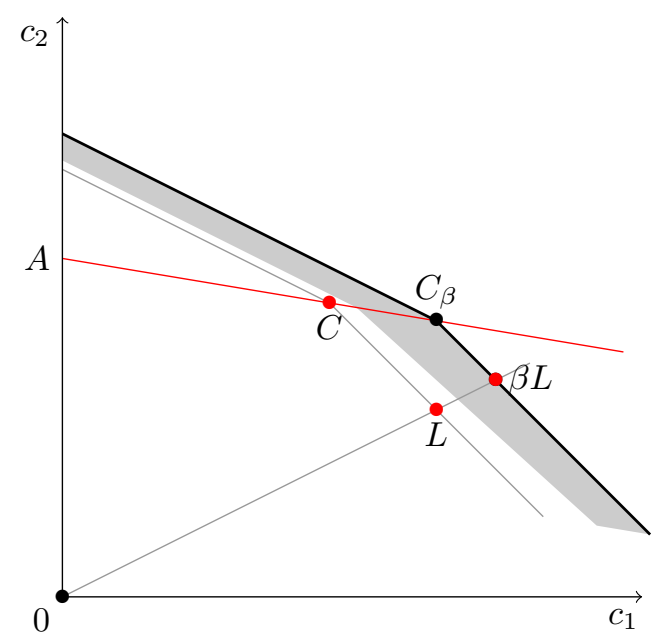

Figure 2: The choice between $K$ and $\beta L$

To complete the proof of proposition 1 we have to show that the allocation $C \in \mathcal{A}$ can be supported at equilibrium.

Lemma 3 If $u(c)=c^{\gamma}$, with $\gamma \in(0,1)$, then the players' behaviors described in $(i)-(i v)$ constitute a subgame perfect equilibrium of the game played amongst the $N$ intermediaries in the presence of a single agent. The corresponding equilibrium allocation will be $C \in \mathcal{A}$.

The proof, developed in the appendix, mainly analyzes the properties of intermediaries' deviations trying to induce $e=a$ as the optimal effort choice. It is shown that following any deviation inducing an increase in the aggregate level of intermediaries' profits, the consumer has an incentive to select $e=b$, by point 2 of lemma 2 . This makes the deviation non-profitable.

In a next step, we look at deviations by latent intermediaries who try to undercut on intermediary 1 's offer. In particular, we have to consider the situation where the consumer trades only with the deviating intermediary at the deviation stage. The deviation is profitable as long as $e=a$ is chosen, and this results in a level of intermediaries profits vis a vis the equilibrium one. However, it is a main implication of point 3 of lemma 2 that the consumer will always take the effort $e=b$ following this kind of deviations.

Finally, one should observe that, being the equilibrium price of the latent contracts (strictly) smaller than the equilibrium one, the only way to profitably deviate inducing $e=b$ as the consumer's optimal choice is to propose a negative insurance contract. We hence prove that none of the intermediaries can unilaterally gain offering one of such contracts, since the agent will have an incentive to select $e=a$, which makes the deviation non profitable. 
We now relate in greater detail our example to the BG work. BG's main result is presented in their proposition 2 , which is stated as follows: ${ }^{7}$

If the set of consumption allocations $X=\left\{\left(x_{1}, x_{2}\right) /\left(1-\pi_{a}\right) u\left(x_{1}\right)+\pi_{a} u\left(x_{2}\right)-u\left(\left(1-\pi_{b}\right) x_{1}+\right.\right.$ $\left.\left.\pi_{b}\left(x_{2}\right)\right)-(a-b) \geq 0\right\}$ is non empty, then any pure strategy equilibrium with $e=a$ induces an allocation $C=\left(c_{1}, c_{2}\right)$ such that:

$$
\left(1-\pi_{a}\right) u\left(c_{1}\right)+\pi_{a} u\left(c_{2}\right)-u\left(\left(1-\pi_{b}\right) c_{1}+\pi_{b}\left(c_{2}\right)\right)-(a-b)=0 .
$$

Equation (8) can be interpreted as an indifference condition for the agent between the equilibrium allocation $C$ where $e=a$ is chosen, and another feasible allocation on the 45 degree line, that can be reached buying additional (latent) insurance at the fair price $\frac{1-\pi_{b}}{\pi_{b}}$. In addition, their proposition 3 states that at any high effort equilibrium there will be at least two active intermediaries, ${ }^{8}$ and proposition 4 provides a constrained efficiency result: every high effort equilibrium is third best optimal. ${ }^{9}$ In particular, the feasible set for the social planner in a "third best program" is given by the set $X$, and any third best allocation belongs to its frontier.

The equilibrium we have constructed does not exhibit the properties described by BG. To clarify this, we first remark that if conditions (1) - (7) are satisfied, then (8) will never hold as an equality. That is, our equilibrium construction also involves latent contracts, but they are issued at a price strictly smaller than the fair one. ${ }^{10}$

One should notice that BG explicitly ruled out the possibility of getting equilibria where the price of latent contracts, say $\tau^{L}$, is strictly smaller than $\frac{1-\pi_{b}}{\pi_{b}}$. In this respect, lemma $\mathrm{A} 1$ of their paper argues that every equilibrium of this sort would not be robust to a deviation by an entrant (or by a latent intermediary) offering a negative insurance contract $d^{\prime}$ at a price $p^{\prime} \in\left(\frac{1-\pi_{b}}{\pi_{b}}, \tau^{L}\right)$.

We indeed provide an example where none of the intermediaries can profitably deviate offering such a $d^{\prime}$. Following the deviation, the agent will find optimal to trade $d^{\prime}$ together with the the contract originally offered by the non-deviating intermediary 1 . This induces her to take the effort $e=a$, which lowers the price effectively posted by the deviator, and blocks the deviation. As a matter of fact, this possibility has not been considered by BG in the proof of lemma A1. ${ }^{11}$

Finally, our equilibrium is supported by only one active intermediary, which shows that the condition identified in BG proposition 3 is indeed not necessary. More precisely, we are showing that the existence of two distinct allocations $Y \in \mathcal{A}$ and $Z \in \mathcal{A}$ such that $U(Y)=U(Z)$ is not a necessary condition for getting a high effort equilibrium, contrarily to what BG have argued. ${ }^{12}$

\footnotetext{
${ }^{7}$ See Bisin and Guaitoli (2004), p. 314.

${ }^{8}$ See Bisin and Guaitoli (2004), p. 315.

${ }^{9}$ See Bisin and Guaitoli (2004), p. 319. For a formal definition of the notion of third best optimality see also Kahn and Mookherjee (1998).

${ }^{10}$ To show that we can in principle apply BG proposition 2 , one has to consider the point $F=(f, f)$ on the 45 degree line, with $f=\left(\frac{\tau^{L} l_{1}+l_{2}}{\tau^{L}+1}\right) . F$ belongs to the tangent to the consumer's indifference curve at $L$, and $U(L)>U(F)$, since $F \in \mathcal{B}$. Using (1) and (2), the last inequality can be written as: $\left(1-\pi_{a}\right) u\left(c_{1}\right)+\pi_{a} u\left(c_{2}\right)-(a-b)>u\left(\frac{\tau^{L} c_{1}+c_{2}}{\tau^{L}+1}\right)$. Also, since $\tau^{L}<\frac{1-\pi_{b}}{\pi_{b}}$, we get: $\frac{\tau^{L} c_{1}+c_{2}}{\tau^{L}+1}>\frac{\frac{1-\pi_{b}}{\pi_{b}} c_{1}+c_{2}}{\frac{1-\pi_{b}}{\pi_{b}}+1}=\left(1-\pi_{b}\right) c_{1}+\pi_{b} c_{2}$. We can hence conclude that $\left(1-\pi_{a}\right) u\left(c_{1}\right)+\pi_{a} u\left(c_{2}\right)-(a-b)>u\left(\left(1-\pi_{b}\right) c_{1}+\pi_{b} c_{2}\right)$, guaranteeing that the set $X$ introduced in BG is non-empty. ${ }^{11}$ See Bisin and Guaitoli (2004), p. 326.

${ }^{12}$ See Bisin and Guaitoli (2004), p. 326.
} 


\section{Welfare analysis}

This section shows that the equilibrium allocations characterized in our proposition 1 fail to be third best efficient. That is, a planner offering state-contingent transfers to the single agent can achieve allocations that Pareto-dominate them.

Let us start by remarking that if exclusivity cannot be enforced, then the intermediaries' strategic interactions must be considered an additional constraint for the planner. In the usual second best perspective the planner retains control on the consumption/insurance allocations, but is subject to the same informational problems as intermediaries in terms of the agent's effort choices. In a non-exclusive context, the planner looses control on intermediaries' behaviors, hence (indirectly) on the total insurance the agent has available for purchases. That is, the planner does not control the effort and the portfolio choices (i.e. the array of $\lambda_{i} \mathrm{~s}$ ) of the agent either.

To properly represent this sort of interactions we consider an alternative formulation of the game, with the planner being an additional intermediary, say intermediary 0 . We also let $d^{0}=\left(d_{1}^{0}, d_{2}^{0}\right)$ be the statecontingent transfer offered by the planner to the single agent. Such a transfer effectively changes the agent's endowment point, and may hence equivalently be thought of as compulsory public insurance. Importantly, the intermediary 0 behaves in a non-strategic way. For every given $d^{0}$, we say that a consumption allocation is third best optimal if the corresponding players' behaviors constitute a best reply to the decision of intermediary $0 .{ }^{13}$

As a starting point, we consider the set of all consumption allocations satisfying (1) and (2), where $\tau^{L} \leq \frac{\left(1-\pi_{b}\right) u^{\prime}\left(l_{1}\right)}{\pi_{b} u^{\prime}\left(l_{2}\right)}$.

Figure 3 depicts the locus of $C$ allocations such that the corresponding $(C, L) \in \mathcal{A} \times \mathcal{B}$ satisfy $(1)$ and (2) for different values of the parameter $\tau^{L}$. We denote $T^{1}$ and $T^{2}$ the locus corresponding to $\tau^{L}=\tau^{1}$ and to $\tau^{L}=\tau^{2}$, respectively.

The next lemma argues that $T$ curves can be ordered according to the parameter $\tau^{L}$. That is, any point of the $T^{2}$ curve is connected by a positive insurance contract to (at least) one point of the $T^{1}$ curve as long as $\tau^{2}<\tau^{1}$.

Lemma $4 T$ curves corresponding to different $\tau$ will not intersect in the $\left(c_{1}, c_{2}\right)$ plane. In addition, any curve $T^{\tau^{\prime}}$ will always lie to the left of the curve $T^{\tau}$ whenever $\tau^{\prime}<\tau$.

We let $T^{0}$ be the $T$ curve corresponding to $\tau^{L}=\frac{1-\pi_{b}}{\pi_{b}}$. In this case, the relevant allocation $L$ belongs to the 45 degree line, which guarantees that the the equation of the $T^{0}$ curve is:

$$
\left(1-\pi_{a}\right) u\left(c_{1}\right)+\pi_{a} u\left(c_{2}\right)-u\left(\left(1-\pi_{b}\right) c_{1}+\pi_{b} c_{2}\right)-(a-b)=0
$$

BG identify the $T^{0}$ curve with the third best frontier. We show in the remaining of the section that any allocation identified in proposition 1 is dominated by an allocation on the $T^{0}$ curve. This indeed establishes constrained inefficiency of market equilibria.

Take any of the $(C, L)$ arrays identified in proposition 1. Consider the array $\left(C^{0}, L^{0}\right)$, with $C^{0} \in T^{0}$, lying on the same indifference curve as $(C, L)$. Clearly, $\left(C^{0}, L^{0}\right)$ is defined in a unique way.

\footnotetext{
${ }^{13}$ This representation is indeed equivalent to considering the following situation: a planner subject to informational and portfolio constraints proposes transfers; given the transfers, the game is effectively played. The feasible set of such a planner is therefore constituted of the set of (subgame perfect) equilibria with transfers of the original game.
} 


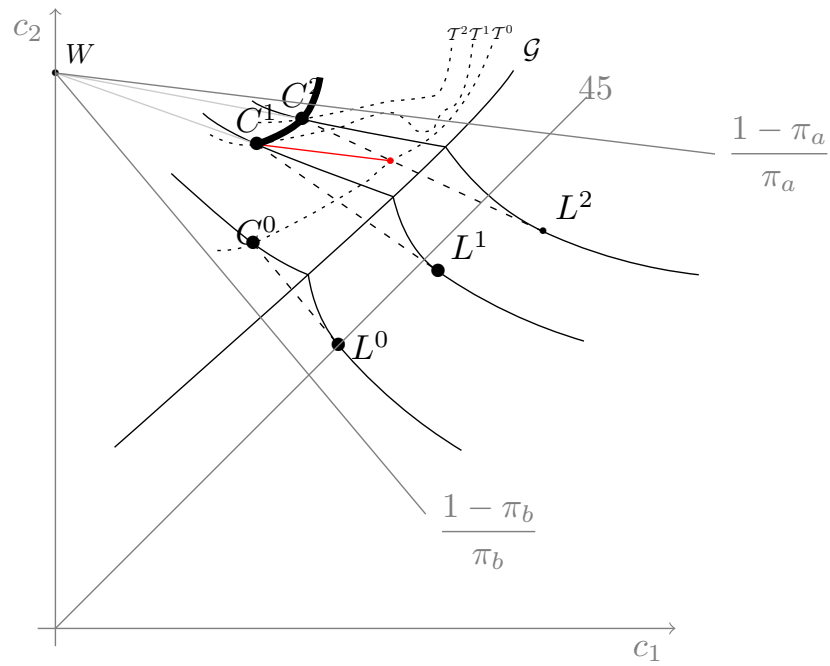

Figure 3: Equilibrium allocations and the price of latent contracts $\tau^{L}$

By construction, $l^{0}=\left(1-\pi_{b}\right) c_{1}^{0}+\pi_{b} c_{2}^{0}$, and

$$
\begin{array}{ccccc}
c_{1} & <c_{1}^{0} & <l^{0} & <l_{1} \\
c_{2} & >c_{2}^{0}>l^{0} & >l_{2} \\
\tau^{L^{0}} & >\tau^{L}>\tau^{C} & >\tau^{C^{0}} .
\end{array}
$$

We then consider the following behaviors:

- The planner proposes the transfers' array $\tilde{d}^{0}=C^{0}-W$ to the single agent; the price of the contract $\tilde{d}^{0}$ is $p^{0}>p^{1}>\frac{1-\pi_{a}}{\pi_{a}}$.

- At least two intermediaries propose the (latent) insurance contract $\tilde{d}^{L}=L^{0}-C^{0}$ at a price $p_{L}=\frac{1-\pi_{b}}{\pi_{b}}$, and all the others offer the null vector $(0,0)$.

- The agent accepts the offer $\tilde{d}^{0}$ and rejects all the other ones. In addition, she selects the high level of effort.

These behaviors support the allocation $C^{0} \in T^{0}$ as an equilibrium with transfers of our game. Importantly, the same allocation could be supported by allowing the planner to propose the non-linear menu $\mathcal{M}^{0}=\left(d^{0}+\lambda \tilde{d}^{0}\right)$, and all intermediaries posting $(0,0)$. The agent selects an element in $\mathcal{M}^{0}$ by choosing a value of $\lambda$ in $[0,1]$.

The following proposition emphasizes that this allocation can indeed be implemented by the planner:

Proposition 2 If the planner provides [public] insurance to the agent through the state-contingent transfers $\tilde{d}^{0}$, then it is a best reply for (at least) two intermediaries to offer the insurance contract $\tilde{d}^{L}$ and for all the others to offer $(0,0)$. The agent will then find optimal not to trade $\tilde{d}^{L}$ and to take the high level of effort.

It is immediate to verify that the profit associated to the $\tilde{d}^{0}$ contract is strictly greater than that of $d^{1}$ whenever the agent sets $e=a$. The inefficiency of the $C$ allocation follows once one observes that the agent may get the same utility at $C$ and at $C^{0}$ while aggregate profit are strictly higher. 


\section{Discussion}

A salient feature of our construction is that every latent contract implies negative virtual profits for the issuer; that is, its price is smaller than the fair price under low effort. Importantly, BG conjecture that any pure strategy equilibrium with negative latent profits would never be robust to deviations from latent intermediaries. As we already said, the problem in their proof appears when they argue that every deviation to a negative insurance contract by any latent intermediary would induce the effort choice $e=b$. We have shown in contrast that, in the context of our example, the consumer will take the opportunity of the negative insurance contract to reach a better allocation in $\mathcal{A}$, which makes the deviation unprofitable.

We now briefly discuss the notion of negative virtual profits for the latent intermediaries. Let us first remark that any $C=\left(c_{1}, c_{2}\right)$ allocation described in our proposition 1 can be as well supported as a symmetric equilibrium of a simpler game where two competitors (principals) can offer any possible subset of alternatives (menus) to the single agent. Instead of thinking of (latent) intermediaries who are offering their contracts anticipating that they will not be accepted and that they will (eventually) incur a loss, one can then represent latent offers as a part of a nonlinear menu proposed by a single competitor (principal). In this last case, every principal issues these additional offers to (strategically) protect his own rents from his rivals' opportunistic behaviors. This provides a further rationale for the relevance of equilibria with virtual negative profits. An extensive analysis of the relationship between latent contracts in competitive economies and menus equilibria is presented in Attar, Campioni, Chassagnon, and Rajan (2008). We discuss in the appendix the general features of this alternative game form and we provide the main intuitions for our result. Interestingly, equilibria of this sort have been intensively examined in the recent literature on games with multiple principals. The possibility to sustain outcomes through offers which are not accepted at equilibrium but are strategically issued by competitors is indeed at the root of the failure of the Revelation Principle in games with multiple principals. ${ }^{14}$ That is, the equilibrium allocation we supported could not have been implemented by letting the two principals compete over singleton contracts. Although most of the recent literature on Common Agency games with moral hazard has typically restricted attention to situations where principals compete through takeit or leave-it offers, there is indeed a very weak foundation for such a choice (see, among others, Attar, Piaser, and Porteiro (2007) and Attar, Majumdar, Piaser, and Porteiro (2008)).

One should also observe that these sort of equilibrium outcomes cannot be straightforwardly eliminated by the introduction of perfection and/or trembling hand requirements. In the terminology of Simon and Stinchombe (1995) we are studying a continuum extensive form game, i.e. a game where at least one agent conditions her actions on previous choices taken in a continuum. To the best of our knowledge, no standard refinement concept has been formulated in such a context. Understanding under which conditions the general treatment of Simon and Stinchombe (1995) extends to this framework is a major topic for future research.

Our analysis therefore suggests that equilibria can be Pareto-ranked according to the price of latent contracts. In particular, those allocations (if there is any) supported by latent insurance offered at a fair price under low effort turn out to be (constrained) efficient. Thus, even though there is no general argument to get rid of these outcomes, it is remarkable that a social planner would never have any incentive to select them.

\footnotetext{
${ }^{14}$ See, among many others, Martimort and Stole (2002).
} 


\section{Appendix}

- Proof of Lemma 1

We first fix $\gamma=1 / 2, C=(25,50)$ and $\tau^{L}=1$. Then, we consider all the solutions such that $w_{1}=0$ and $w_{2}=$ $+\left(\tau^{C}-0.05\right) c_{1}+c_{2}$. For $(2)$ and $(5)$ to be verified, one has to impose $l_{1}=c_{1}+\frac{\left(\tau^{C}-0.05\right) c_{1}+c_{2}}{2\left(1+\tau^{L}\right)}$ and $l_{2}=$ $c_{2}-\tau^{L} \frac{\left(\tau^{C}-0.05\right) c_{1}+c_{2}}{2\left(1+\tau^{L}\right)}$. The corresponding values of $\pi_{a}$ and $\pi_{b}$ are identified by the relationships $M R S(C)=$ $\tau^{C}$ and $\operatorname{MRS}(L)=\tau^{L}: \quad \pi_{a}=\frac{\sqrt{c_{2}}}{\tau^{C} \sqrt{c_{1}}+\sqrt{c_{2}}}, \pi_{b}=\frac{\sqrt{l_{2}}}{\tau^{L} \sqrt{l_{1}}+\sqrt{L_{2}}}$. Then, (3) can be rewritten as:

$$
\tau^{c}\left(\sqrt{l_{1}}+\sqrt{l_{2}}\right)\left(\sqrt{c_{2}}-\sqrt{c_{1}}\right)=c_{2}-\left(\tau^{C}\right)^{2} c_{1},
$$

where we used the fact that $\tau^{L}=1$. This equation has two solutions in $\tau^{C}$, a positive and a negative one. We select the positive one so to get $\tau^{c} \approx 0.996199 .{ }^{15}$ Given $\tau^{C}$, from $(2)$ and $(5)$ we derive: $L \approx(43.4137,31.5863)$, and one has to notice that since $l_{1}>l_{2}$, we directly have that $L \in \mathcal{B}$. Finally, the parameter $a-b$ is chosen so to satisfy (1). In particular, we get $a-b \approx 0.0721456$. It is then possible to verify by direct inspection that all inequalities in (4), (6) and (7) are verified and that $C \in \mathcal{A} .^{16}$

\section{- Proof of Lemma 2}

1. We first remark that if $C_{\alpha} \in \mathcal{B}$, then one immediately gets $U\left(C_{\alpha}\right)<U(\alpha L)$, since $\alpha L$ is the optimal consumption choice when $e=b$ is taken and that $C_{\alpha}$ and $\alpha L$ are connected by a line of slope $-\tau^{L}$. We therefore consider in what follows the case $C_{\alpha} \in \mathcal{A}$. In this situation, $\frac{d}{d \alpha} U\left(C_{\alpha}\right)=\pi_{a}(1-$ $\left.\pi_{a}\right) f^{\prime}(\alpha)\left(u^{\prime}\left(c_{1 \alpha}\right)-u^{\prime}\left(c_{2 \alpha}\right)\right)$. One should notice that for $\alpha \geq(\leq) 1, \frac{d}{d \alpha} U\left(C_{\alpha}\right)$ is bounded above (below) by $\pi_{a}\left(1-\pi_{a}\right) \alpha^{\gamma-1} f^{\prime}(\alpha)\left(u^{\prime}\left(c_{1}\right)-u^{\prime}\left(c_{2}\right)\right)$, since $u^{\prime}\left(c_{1 \alpha}\right)$ is smaller (greater) than $u^{\prime}\left(\alpha c_{1}\right)$. Furthermore, $\alpha L$ is always below the 45 degree line and $U(\alpha L)=\left(1-\pi_{b}\right) u\left(l_{1} \alpha\right)+\pi_{b} u\left(l_{2} \alpha\right)-b$ has the following derivative:

$$
\begin{aligned}
\frac{d}{d \alpha} U(\alpha L) & =\alpha^{\gamma-1}\left(\left(1-\pi_{b}\right) l_{1} u^{\prime}\left(l_{1}\right)+\pi_{b} l_{2} u^{\prime}\left(l_{2}\right)\right) \\
& =\alpha^{\gamma-1} \pi_{b} u^{\prime}\left(l_{2}\right)\left(\tau^{L} l_{1}+l_{2}\right) \\
& =\alpha^{\gamma-1} \pi_{b} u^{\prime}\left(l_{2}\right)\left(f^{\prime}(\alpha) \pi_{a}\left(\tau^{L}-\tau^{a}\right)\right) \\
& =\alpha^{\gamma-1} f^{\prime}(\alpha)\left(\pi_{a}\left(1-\pi_{b}\right) u^{\prime}\left(l_{1}\right)-\left(1-\pi_{a}\right) \pi_{b} u^{\prime}\left(l_{2}\right)\right),
\end{aligned}
$$

where the last two equalities have been derived using (2), (3) and the definition of $f(\alpha)$ provided in footnote 6 .

Then, for $\alpha \geq 1(\alpha \leq 1), \frac{d}{d \alpha}\left[U\left(C_{\alpha}\right)-U(\alpha L)\right]$ is bounded above (below) by $\alpha^{\gamma-1} f^{\prime}(\alpha)\left(\pi_{a}(1-\right.$ $\left.\left.\pi_{a}\right)\left(u^{\prime}\left(c_{1}\right)-u^{\prime}\left(c_{2}\right)\right)-\left(\pi_{a}\left(1-\pi_{b}\right) u^{\prime}\left(l_{1}\right)-\left(1-\pi_{a}\right) \pi_{b} u^{\prime}\left(l_{2}\right)\right)\right)$ which is zero by $(3)$. Hence, $U\left(C_{\alpha}\right)-$ $U(\alpha L)$ has a maximum in $\alpha=1$. If $\alpha=1, U\left(C_{\alpha}\right)-U(\alpha L)=U(C)-U(L)=0$ from (1).

2. We first remark that if $K \in \mathcal{B}$, then one immediately gets $U(K)<U(\alpha L)$, since $\alpha L$ is the optimal consumption choice when $e=b$ is taken and $K$ and $\alpha L$ are connected by a line of slope $-\tau^{L}$. We henceforth restrict to the case $K \in \mathcal{A}$. Let us first take $\alpha_{0}$ to be the value of $\alpha$ such that $M R S\left(C_{\alpha_{0}}\right)=\tau^{L}$. For $\alpha \geq \alpha_{0}$, one can directly verify that $M R S\left(C_{\alpha}\right) \leq \tau^{L}$. It is then immediate that $U(K)<U\left(C_{\alpha}\right)$ for every $K \in \mathcal{A}$ belonging to the segment (of slope $\tau^{L}$ ) that connects $C_{\alpha}$ and $\alpha L$. It follows from part 1 of the lemma that $U(K)<U(\alpha L)$. For $\alpha<\alpha_{0}, M R S\left(C_{\alpha}\right)>\tau^{L}$, and the agent's consumption choice on

\footnotetext{
${ }^{15}$ The solution has been calculated using the NSolve function in Mathematica. The code is available from the authors.

${ }^{16}$ This has been established running a standard test in Mathematica.
} 
the line passing through $C_{\alpha}$ and $\alpha L$ will be $\frac{\alpha}{\alpha_{0}} C_{\alpha_{0}} \in \mathcal{A}$. Using again point 1 of the lemma we get, after some computations:

$$
U(K)-U(\alpha L) \leq U\left(\frac{\alpha}{\alpha_{0}} C_{\alpha_{0}}\right)-U(\alpha L)=\left(\frac{\alpha}{\alpha_{0}}\right)^{\gamma}\left(U\left(C_{\alpha_{0}}\right)-U\left(\alpha_{0} L\right)\right)+(a-b)\left(\left(\frac{\alpha}{\alpha_{0}}\right)^{\gamma}-1\right)<0
$$

3. We first remark that if $K \in \mathcal{B}$, then one immediately gets $U(K)<U(\beta L)$, since $K$ is below the line of slope $-\tau^{L}$ passing through $\beta L$. Take any $\beta$ and $C_{\beta}$ satisfying the conditions stated in the Lemma. We first let $N=\left(n_{1}, n_{2}\right)$ be the consumption allocation lying on the line of slope $-\tau^{a}=-\frac{1-\pi_{a}}{\pi_{a}}$ passing through $C$ and such that $\frac{\left(1-\pi_{a}\right) u^{\prime}\left(n_{1}\right)}{\pi_{a} u^{\prime}\left(n_{2}\right)}=p_{1}$.

In a first step, we consider the situation where $N$ lies at the right of $C_{\beta}$, which corresponds to the case where $\frac{\left(1-\pi_{a}\right) u^{\prime}\left(c_{\beta 1}\right)}{\pi_{a} u^{\prime}\left(c_{\beta 2}\right)} \geq p_{1}$.

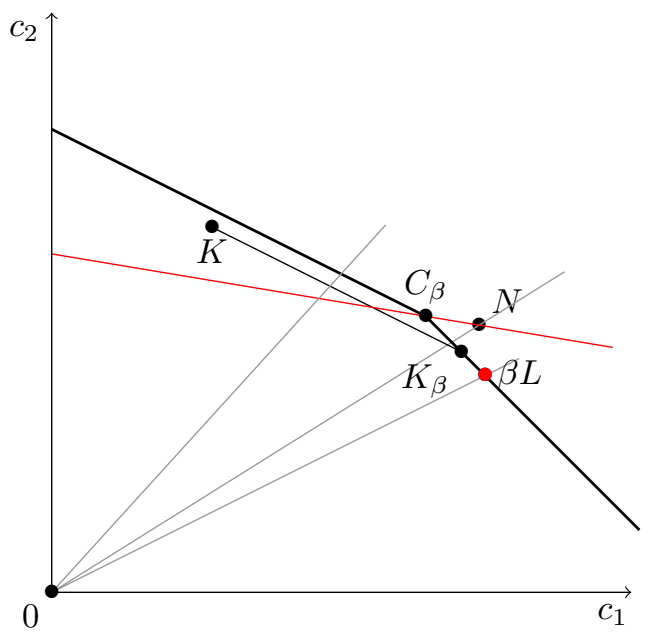

Figure 4: The choice between $K, C_{\beta}$ and $\beta L$

To get the result, one should consider the allocation $K_{\beta}=\left(k_{\beta 1}, k_{\beta 2}\right)$, at the intersection of the line of slope $-p_{1}$ passing through $K$ and the line connecting $C_{\beta}$ and $\beta L$, which has slope $-\tau^{L}$ (see Figure 4 ). It is then immediate to show that the consumer will always prefer $\beta L$ to $K$ :

- If $\frac{\left(1-\pi_{a}\right) u^{\prime}\left(k_{\beta 1}\right)}{\pi_{a} u^{\prime}\left(k_{\beta 2}\right)} \geq p_{1}$, then $U(K)<U\left(K_{\beta}\right)<U(\beta L)$. The first inequality follows from the fact that, in such a case, the agent's utility is increasing at the left of $K_{\beta}$, while the second one is an implication of point 2 of this Lemma.

- If $\frac{\left(1-\pi_{a}\right) u^{\prime}\left(k_{\beta 1}\right)}{\pi_{a} u^{\prime}\left(k_{\beta 2}\right)}<p_{1}$, which is the case considered in Figure 4, then one directly gets: $U(K)<$ $U\left(\alpha^{*} N\right)<U(N)<U(\beta L)$. The first inequality comes from the fact that $\alpha^{*} N$ is the allocation on the line connecting $K$ and $K_{\beta}$ satisfying $\frac{\left(1-\pi_{a}\right) u^{\prime}\left(\alpha^{*} n_{1}\right)}{\pi_{a} u^{\prime}\left(\alpha^{*} n_{2}\right)}=p_{1}$, the second one is implied by $\alpha^{*}<1$, and the last one is obtained using point 2 of this Lemma.

In a next step, we consider the situation where $N$ lies between $C$ and $C_{\beta}$, that is: such that $\frac{\left(1-\pi_{a}\right) u^{\prime}\left(n_{1}\right)}{\pi_{a} u^{\prime}\left(n_{2}\right)}<$ $p_{1}$. Let us first take the particular $\alpha$ such that $\alpha N$ lies on the line of slope $p_{1}$ passing through $C_{\beta}$. Necessarily, $\alpha>1$, since $p_{1}>\tau^{a}$. By construction, we have that $U(K) \leq \tilde{U}(\alpha N, a)$. That is, the utility that the 
consumer can achieve by taking $e=a$ at the deviation stage is always dominated by that associated to the choice of the allocation $\alpha N$ together with $e=a .{ }^{17}$ To establish this point of the lemma, it will therefore suffice to show that $\tilde{U}(\alpha N, a) \leq U(\beta L)$. We will prove this inequality in a direct way.

We first recall that $C_{\beta}=\left(c_{1}+(\beta-1) \frac{\tau^{L} c_{1}+c_{2}}{\tau^{L}-\tau^{a}}, c_{2}-\tau^{a}(\beta-1) \frac{\tau^{L} c_{1}+c_{2}}{\tau^{L}-\tau^{a}}\right) .{ }^{18}$ Then, since $\alpha N$ and $C_{\beta}$ are connected by a line of slope $p_{1}$, one gets:

$$
\begin{aligned}
\alpha\left(p_{1} n_{1}+n_{2}\right) & =\left(p_{1} c_{1}+c_{2}\right)+(\beta-1) \frac{\tau^{L} c_{1}+c_{2}}{\tau^{L}-\tau^{a}}\left(p_{1}-\tau^{a}\right) \\
(\alpha-1)\left(p_{1} n_{1}+n_{2}\right) & =-\left(p_{1}-\tau^{a}\right)\left(n_{1}-c_{1}\right)+(\beta-1) \frac{\tau^{L} c_{1}+c_{2}}{\tau^{L}-\tau^{a}}\left(p_{1}-\tau^{a}\right) \\
(\alpha-1) & =-\frac{\left(p_{1}-\tau^{a}\right)\left(n_{1}-c_{1}\right)}{p_{1} n_{1}+n_{2}}+b(\beta-1) \\
\frac{\alpha}{b \beta} & =1+\frac{1-a-b}{b \beta}
\end{aligned}
$$

with $a=\frac{\left(p_{1}-\tau^{a}\right)\left(n_{1}-c_{1}\right)}{p_{1} n_{1}+n_{2}}$ and $b=\frac{\tau^{L} c_{1}+c_{2}}{\tau^{L}-\tau^{a}} \frac{p_{1}-\tau^{a}}{p_{1} n_{1}+n_{2}}$.

One should observe that since $-\tau^{a}\left(n_{1}-c_{1}\right)=\left(n_{2}-c_{2}\right)$, then:

$$
\begin{aligned}
a+b & =\frac{\left(p_{1}-\tau^{a}\right)}{\left(\tau^{L}-\tau^{a}\right)\left(p_{1} n_{1}+n_{2}\right)}\left[\left(\tau^{L}-\tau^{a}\right)\left(n_{1}-c_{1}\right)+\tau^{L} c_{1}+c_{2}\right] \\
& =\frac{\left(p_{1}-\tau^{a}\right)}{\left(\tau^{L}-\tau^{a}\right)\left(p_{1} n_{1}+n_{2}\right)}\left[\tau^{L}\left(n_{1}-c_{1}\right)+\left(n_{2}-c_{2}\right)+\tau^{L} c_{1}+c_{2}\right] \\
& =\frac{\frac{p_{1}-\tau^{a}}{p_{1} n_{1}+n_{2}}}{\tau^{L}-\tau^{a}} \leq 1, \\
\tau^{L} n_{1}+n_{2} &
\end{aligned}
$$

which implies that

$$
\frac{\alpha}{\beta} \geq b>0 .
$$

Equations (11)-(15) reformulate the relationship between $\alpha N$ and $C_{\beta}$ in terms of the variables $\alpha$ and $\beta$. In particular, equation (15) puts a lower bound on the ratio between $\alpha$ and $\beta$.

Then, consider the difference:

$$
\left.\left.U(\beta L)-\tilde{U}(\alpha N, a)=\left[\left(1-\pi_{b}\right) u\left(\beta l_{1}\right)+\pi_{b} u\left(\beta l_{2}\right)-b\right)\right]-\left[\left(1-\pi_{a}\right) u\left(\alpha n_{1}\right)+\pi_{a} u\left(\alpha n_{2}\right)-a\right)\right] .
$$

Since (14) defines an implicit relationship between $\alpha$ and $\beta$, we can write $g(\beta)=U(\beta L)-\tilde{U}(\alpha N, a)$ which is a continuous and differentiable function. Its derivative is:

$$
\begin{aligned}
g^{\prime}(\beta) & =\left[\left(1-\pi_{b}\right) u^{\prime}\left(l_{1}\right) l_{1}+\pi_{b} u^{\prime}\left(l_{2}\right) l_{2}\right] \beta^{\gamma-1}-\left[\left(1-\pi_{a}\right) u^{\prime}\left(n_{1}\right) n_{1}+\pi_{a} u^{\prime}\left(n_{2}\right) n_{2}\right] \alpha^{\gamma-1} b \\
& =\pi_{b} u^{\prime}\left(l_{2}\right)\left(\tau^{L} l_{1}+l_{2}\right) \beta^{\gamma-1}-\pi_{a} u^{\prime}\left(n_{2}\right)\left(p_{1} n_{1}+n_{2}\right) \alpha^{\gamma-1} b \\
& =\frac{\tau^{L} c_{1}+c_{2}}{\tau^{L}-\tau^{a}}\left[\beta^{\gamma-1}\left(\pi_{b} u^{\prime}\left(l_{2}\right)\left[\tau^{L}-\tau^{a}\right]\right)-\alpha^{\gamma-1}\left(\pi_{a} u^{\prime}\left(c_{2}\right)\left[\tau^{C}-\tau^{a}\right]\right) \frac{p_{1}-\tau^{a}}{\tau^{C}-\tau^{a}} \frac{u^{\prime}\left(n_{2}\right)}{u^{\prime}\left(c_{2}\right)}\right] .
\end{aligned}
$$

We remark here that equation (3) can be rewritten as $\pi_{b} u^{\prime}\left(l_{2}\right)\left[\tau^{L}-\tau^{a}\right]=\pi_{a} u^{\prime}\left(c_{2}\right)\left[\tau^{C}-\tau^{a}\right]$. Then, after simple manipulations, one gets:

$$
\frac{g^{\prime}(\beta) \alpha^{1-\gamma}}{\pi_{b} u^{\prime}\left(l_{2}\right)\left(\tau^{L} c_{1}+c_{2}\right)}=\left(\frac{\alpha}{\beta}\right)^{1-\gamma}-\frac{p_{1}-\tau^{a}}{\tau^{C}-\tau^{a}}\left(\frac{n_{2}}{c_{2}}\right)^{\gamma-1} .
$$

\footnotetext{
${ }^{17}$ One should observe that we are not claiming that the allocation $\alpha N$ belongs to $\mathcal{A}$. We are only interested in the utility level $\tilde{U}(\alpha N, a)$.

${ }^{18}$ See footnote 6
} 
Now, using inequality (15), we obtain after further manipulations that:

$$
\frac{g^{\prime}(\beta) \alpha^{1-\gamma}}{\pi_{b} u^{\prime}\left(l_{2}\right)\left(\tau^{L} c_{1}+c_{2}\right)}\left(\frac{\tau^{C}-\tau^{a}}{p_{1}-\tau^{a}}\right)^{1-\gamma}\left(\frac{n_{2}}{c_{2}}\right)^{1-\gamma} \geq\left(\frac{\tau^{L} c_{1}+c_{2}}{c_{2}} \frac{n_{2}}{p_{1} n_{1}+n_{2}} \frac{\tau^{C}-\tau^{a}}{\tau^{L}-\tau^{a}}\right)^{1-\gamma}-\left(\frac{p_{1}-\tau^{a}}{\tau^{C}-\tau^{a}}\right)^{\gamma} .
$$

Now since $\frac{p_{1} n_{1}+n_{2}}{n_{2}}=1+\tau^{a}\left(\frac{n_{1}}{n_{2}}\right)^{\gamma} \leq 1+\tau^{a}=\frac{1}{\pi_{a}}$ and using equation (7), one can conclude that $g^{\prime}(\beta)>0$.

We remark that the smallest $\beta$ compatible with the condition $\frac{\left(1-\pi_{a}\right) u^{\prime}\left(c_{\beta 1}\right)}{\pi_{a} u^{\prime}\left(c_{\beta 2}\right)} \leq p_{1}$ is such that $C_{\beta}=N$. From equation (13) this is obtained when $\alpha=1$ and $\beta=1+\frac{a}{b}$. It hence follows from point 1 of this lemma that $U(\beta L)>U\left(C_{\beta}\right)=U(N) \geq \tilde{U}(N, a)$, which corresponds to $g\left(1+\frac{a}{b}\right)>0$. Concluding, we have that $g(\beta)>0$, for every $\beta>1$ and such that $\frac{\left(1-\pi_{a}\right) u^{\prime}\left(c_{\beta 1}\right)}{\pi_{a} u^{\prime}\left(c_{\beta 2}\right)} \leq p_{1}$.

\section{- Proof of Lemma 3}

Let us first examine the agent's behavior. Then, $C=\left(c_{1}, c_{2}\right)$ is (weakly) preferred to any of the allocations belonging to the frontier of the feasible (consumption) set. It will hence be a best reply for the agent to accept the contract of intermediary 1 and reject all the others.

Then, we consider intermediaries' behaviors. It is sufficient to restrict the analysis to deviations in take-it or leave-it offers.

Let us consider intermediary 1 . We first examine the deviations $d^{1 \prime}$ inducing a high level of effort and we take $K \in \mathcal{A}$ to be the optimal consumption choice of the agent at the deviation stage. If $d^{1 \prime}$ is a negative insurance contract, to guarantee positive profits, its price should be below $\frac{1-\pi_{a}}{\pi_{a}}$, since the agent will select $e=a$. Then, whenever such a $d^{1 \prime}$ is bought, the corresponding $K$ should lie to the left of the line connecting $W$ and $D$ (see Figure 1), which contradicts the fact that $K$ is optimally chosen.

If $d^{1 \prime}$ is a positive insurance contract, to guarantee that the deviation is profitable, it must be that $-\vec{\pi}_{a} \cdot d^{1 \prime}>$ $-\vec{\pi}_{a} \cdot d^{1}$. It hence follows from (6) that $-\vec{\pi}_{a} \cdot d^{1 \prime}>-\vec{\pi}_{a} \cdot d^{2}$. Moreover, $d^{1 \prime}$ cannot be offered at a price (strictly) greater than $\tau^{L},{ }^{19}$ and this implies $d^{1 \prime}=d^{2}+d^{1 \prime \prime}$, with $d^{1 \prime \prime}$ being a positive insurance contract which price is smaller than $\tau^{L}$. The terminal point on the frontier of feasible consumptions corresponding to the offers $\left(d^{1 \prime}, d^{2}, \ldots, d^{J}\right)$, i.e. $W+d^{1 \prime \prime}+3 d^{2},{ }^{20}$, will hence fall below the line passing through the origin and $L$, as it is the case for the point $W+3 d^{2} \cdot{ }^{21}$ At the deviation stage, the agent can therefore achieve her optimal choice in the subset $\mathcal{B}$, since it lies on the line passing through the origin and $L$. Thus, if one takes the particular $\alpha \in \mathbb{R}_{+}$such that $C_{\alpha}, K$ and $\alpha L$ are on the same line, then $K \in \mathcal{A}$ will be between $C_{\alpha}$ and $\alpha L$. Hence, lemma 2 implies that $U(K)<U(\alpha L)$, that constitutes a contradiction, since $\alpha L$ is available. ${ }^{22}$

Intermediary 1 cannot profitably deviate inducing low effort either. A deviation to a positive insurance contract inducing $e=b$ could be profitable only if the price is greater than $\frac{1-\pi_{b}}{\pi_{b}}$. The agent, though, will never have any incentive to buy such a contract since the optimal available allocation when $d^{1 \prime}$ is not accepted is a point like $D$ on the 45 degree line (see Figure 1). If $d^{1 \prime}$ is a negative insurance contract, the relevant price should be greater than $\tau^{L}$, for the agent to have an incentive to buy it, and smaller than $\frac{\left(1-\pi_{b}\right)}{\pi_{b}}$, to be profitable for the deviator. Since the agent can always achieve the allocation $D$ by rejecting $d^{1 \prime}$, the deviating contract will never be bought.

We now consider the behavior of intermediary 2 (and 3) and first show that there is no profitable deviation inducing $e=a$. We keep denoting $K \in \mathcal{A}$ the optimal consumption choice of the agent, given the deviation $d^{2 \prime}$ of

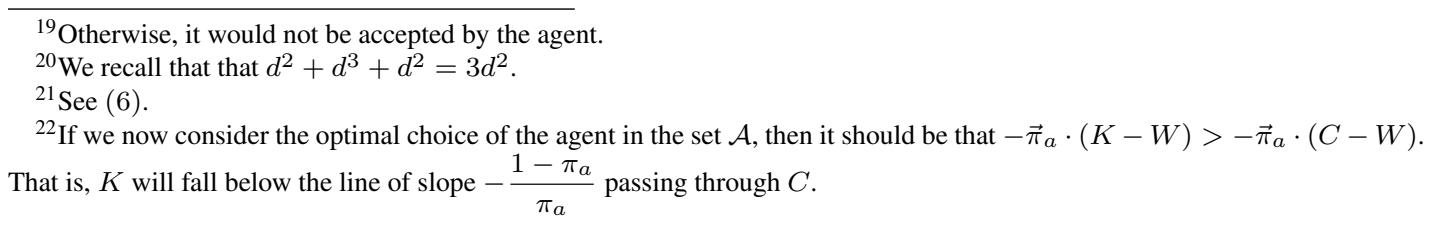


intermediary 2. If $d^{2 \prime}$ is a positive insurance contract, ${ }^{23}$ and $-\vec{\pi}_{a} \cdot(K-C)>0$, one can use an argument similar to that suggested in the previous paragraph. In this case, the relevant $\alpha$ will be greater than 1 , which implies that the corresponding $\alpha L$ will be available and that lemma 2 can be applied.

The case $-\vec{\pi}_{a} \cdot(K-C) \leq 0$ only takes place when the contract $d^{1}$ is not bought at the deviation stage. ${ }^{24}$ The set of feasible consumption choices corresponding to such a situation is depicted in Figure 5 . For every $K \in \mathcal{A}$, the agent can achieve both $M=K+d^{1}$ and $\beta L$, which lies on the ray connecting the origin to $L{ }^{25}$ We show in the next paragraph that the agent will always prefer the allocation $\beta L$ to $K$, which makes the deviation non profitable.

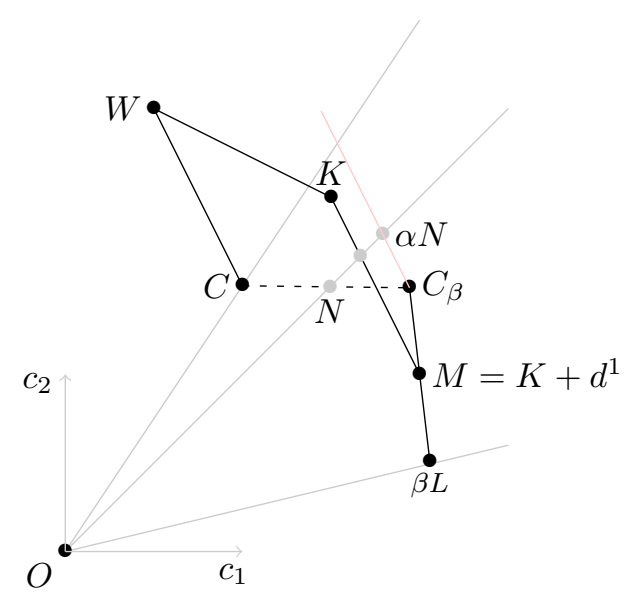

Figure 5: A deviation of intermediary 2

We remark that the inequalities

$$
\tilde{U}(M, a) \leq U(M) \leq U(K)
$$

are always satisfied by construction. As a consequence, $\frac{\left(1-\pi_{a}\right) u^{\prime}\left(m_{1}\right)}{\pi_{a} u^{\prime}\left(m_{2}\right)} \leq p_{1}$.

One should now observe that every allocation $K=\left(k_{1}, k_{2}\right) \in \mathcal{A}$ also has to satisfy the following inequalities:

$$
\begin{gathered}
p_{1} k_{1}+k_{2} \leq p_{1} c_{\beta 1}+c_{\beta 2} \\
\tau^{a} k_{1}+k_{2} \geq \tau^{a} c_{\beta 1}+c_{\beta 2} .
\end{gathered}
$$

In addition, it is immediate to verify that any allocation $K$ satisfying (16) and (17) will be such that $\frac{u^{\prime}\left(k_{1}\right)}{u^{\prime}\left(k_{2}\right)} \geq$ $\frac{u^{\prime}\left(c_{\beta 1}\right)}{u^{\prime}\left(c_{\beta 2}\right)}$.

We hence have to consider two possibilities. First, let suppose that $\frac{\left(1-\pi_{a}\right) u^{\prime}\left(c_{\beta 1}\right)}{\pi_{a} u^{\prime}\left(c_{\beta 2}\right)}>p_{1}$.

Then, the following inequalities will hold:

$$
U(K) \leq \tilde{U}\left(C_{\beta}, a\right) \leq U\left(C_{\beta}\right)<U(\beta L)
$$

where the last relationship follows from point 2 of lemma 2. The deviation will hence induce low effort, and this makes it not profitable.

\footnotetext{
${ }^{23}$ The case of $d^{2 \prime}$ being a negative insurance contract is not of great interest, since the agent will never have an incentive to accept a negative insurance contract issued at a price smaller than $\frac{1-\pi_{a}}{\pi_{a}}$ and select the high effort $e=a$

${ }^{24}$ Whenever $d^{1}$ is bought, then we have that: $-\vec{\pi}_{a} \cdot(K-W) \geq-\vec{\pi}_{a} \cdot d^{1}-\vec{\pi}_{a} \cdot d^{2 \prime}>-\vec{\pi}_{a} \cdot d^{1}=-\vec{\pi}_{a} \cdot(C-W)$.

${ }^{25}$ The availability of $\beta L$ is guaranteed by construction. Indeed, we already know that adding $d^{3}$ to $C$ is enough to achieve $L$. Since $d^{2 \prime}$ is a positive insurance contract, by adding $d^{2 \prime}$ and $d^{3}$ to $C$ we make $\beta L$ available.
} 
Second, consider the case $\frac{\left(1-\pi_{a}\right) u^{\prime}\left(c_{\beta 1}\right)}{\pi_{a} u^{\prime}\left(c_{\beta 2}\right)} \leq p_{1}$. We start by remarking that $-\vec{\pi}_{a} \cdot M>-\vec{\pi}_{a} \cdot C$, since $M=K+d^{1}=C+d^{2 \prime}$. In other words, $M$ falls between $C_{\beta}$ and $\beta L$. As a consequence, one gets that $p_{1} m_{1}+m_{2} \leq p_{1} c_{\beta 1}+c_{\beta 2}$ which follows from $p_{1} \leq \tau^{L}$. However, $p_{1} m_{1}+m_{2}=p_{1} k_{1}+k_{2}$ because $M=K+d^{1}$. Thus, we get $\frac{\left(1-\pi_{a}\right) u^{\prime}\left(c_{\beta 1}\right)}{\pi_{a} u^{\prime}\left(c_{\beta 2}\right)} \leq p_{1}$. This allows us to directly apply point 3 of lemma 2 , so to get the result.

In a next step we investigate whether there is any deviation of intermediary 2 that induces the low effort $e=b$. If $d^{2 \prime}$ is a positive insurance contract, then it must involve a price strictly greater than $\frac{1-\pi_{b}}{\pi_{b}}$ to be profitable. The fact that the consumer can already achieve her optimal choice at a price $\tau^{L}$, guarantees that such a deviation would never be accepted. If $d^{2 \prime}$ is a negative insurance contract, then its price should be (strictly) greater than $\tau^{L}$. The frontier of the set of feasible consumptions will then be the line connecting $W+d^{2 \prime}, C+d^{2 \prime}$ and $L+d^{2 \prime}$. That is, the agent can choose any consumption allocation between the two points $C+d^{2 \prime} \in \mathcal{A}$ and $L+d^{2 \prime} \in \mathcal{B}$ which are connected by a line of slope $\tau^{L}$. Consider now the particular $\alpha>1$ such that $C+d^{2 \prime}, \alpha C$ and $\alpha L$ are on the same line. One should notice that $\alpha C$ is also available to the consumer, given that $C+d^{2 \prime}$ falls over the ray connecting the origin with $C$ (i.e. it is at the north-west of $\alpha C$ ). Since the $M R S(\alpha C)=\tau^{C}<\tau^{L}$, the best consumption allocation available to the agent in the $\mathcal{A}$ subspace will lie at the north-west of $\alpha C$. We take this allocation to be $K$; it hence follows that $U(K)>U(\alpha C)$. In addition, we remark that $U\left(L+d^{2 \prime}\right)<U(\alpha L)$, since $M R S\left(L+d^{2 \prime}\right)>\tau^{L}$. Now, if $u(c)=c^{\gamma}$, then it is straightforward to establish that $U(\alpha C)>U(\alpha L)$ for every $\alpha>1 .^{26}$ Overall, one gets

$$
U(K)>U(\alpha C)>U(\alpha L)>U\left(L+d^{2 \prime}\right)
$$

The deviation to the negative insurance contract $d^{2 \prime}$ will hence induce $e=a$ as the optimal effort choice, which makes it non profitable.

Finally, one has to consider the deviations of all intermediaries offering the null contract $(0,0)$. To show that these deviations will never be profitable, one can use the same argument developed for the latent intermediaries 2 and 3. Indeed, when an intermediary offering a null contract (i.e. an entrant) deviates, the set of feasible allocations for the agent coincides with the set of allocations that were available following the deviation $d^{2 \prime}$ together with some additional amount of insurance available at a price $\tau^{L}$. The agent will hence behave in the same way following a deviation to $d^{2 \prime}$ and following a deviation from any of the entrants. ${ }^{27}$

\section{- Proof of Lemma 4}

Proof. We prove that $T$ curves corresponding to different $\tau$ will not intersect in the $\left(c_{1}, c_{2}\right)$ plane; this guarantees that any $T^{\tau^{\prime}}$ curve always lies to the left of the $T^{\tau}$ curve whenever $\tau^{\prime}<\tau$. Let us first suppose that an intersection point exists, and denote it by $C$. That is, there should be two allocation arrays, say $(C, L)$ and $\left(C, L^{\prime}\right)$, belonging to $T^{\tau}$ and to $T^{\tau^{\prime}}$, respectively. The intersection point $C$ hence satisfies (2) for $\tau^{L}=\tau$ and $\tau^{L}=\tau^{\prime}$. In other words, $C$ is at the intersection of the two lines tangent in $L$ and in $L^{\prime}$, respectively, to the agent's indifference curve in $\mathcal{B}$. It follows that such an intersection point will lie "between" $L$ and $L^{\prime}$, that is:

$$
\begin{aligned}
& \min \left(l_{1}, l_{1}^{\prime}\right) \leq c_{1} \leq \max \left(l_{1}, l_{1}^{\prime}\right) \\
& \min \left(l_{2}, l_{2}^{\prime}\right) \leq c_{2} \leq \max \left(l_{2}, l_{2}^{\prime}\right)
\end{aligned}
$$

However, the conditions above together with the fact that $C, L$ and $L^{\prime}$ belong to the same indifference curve (by (1)) imply that $C \in \mathcal{B}$, a contradiction.

\footnotetext{
${ }^{26}$ We have that $U(\alpha C)-U(\alpha L)=(U(\alpha C)+a)-(U(\alpha L)+b)-(a-b)=\alpha^{\gamma}(U(C)+a)-\alpha^{\gamma}(U(L)+b)-(a-b)=$ $\left(\alpha^{\gamma}-1\right)(a-b)>0$, for $\alpha>1$.

${ }^{27}$ More precisely, when we considered deviations that induce $e=a$, it was enough to prove that the consumer always has an incentive to select an allocation on the ray connecting 0 and $L$. This will of course remain available in this context. All deviations inducing $e=b$ were instead blocked independently of the availability of the optimal consumption choice in $\mathcal{B}$. Clearly, such an optimal choice will not be modified by the availability of additional insurance at a price $\tau^{L}$.
} 
Let us take two points $C \in T^{\tau}$ and $C^{\prime} \in T^{\tau^{\prime}}$ belonging to the same indifference curve. Then, (strict) convexity of preferences on $\mathcal{B}$ implies that $L$ will be at the north-west of $L^{\prime}\left(l_{1}^{\prime}>l_{1}\right.$ and $\left.l_{2}^{\prime}<l_{2}\right)$. Consider now the lines tangent to that indifference curve in $L$ and in $L^{\prime}$, respectively: (2) guarantees that $L$ is connected to $C \in \mathcal{A}$ and $L^{\prime}$ to $C^{\prime} \in \mathcal{A}$. Let $I$ denote the intersection of these two tangents. Clearly, $I \in \mathcal{B}$, and the slopes of the $I C$ and of the $I C^{\prime}$ lines are $\tau$ and $\tau^{\prime}$, respectively. Finally, one has to observe that $C$ and $C^{\prime}$ are on a same convex curve, and $I$ lies below that curve (this follows from the relationship $\tau^{C}<\tau^{L}$ which holds at equilibrium). A simple graphical argument will then imply that if $\tau^{\prime}<\tau$, then $C^{\prime}$ must be at the left of $C\left(c_{1}^{\prime}<c_{1}\right.$ and $\left.c_{2}^{\prime}>c_{2}\right)$. That is, $T^{\tau^{\prime}}$ is in the interior (at the left) of $T^{\tau}$. That is, for any $C$ belonging to a $T^{\tau}$ curve, with $\tau<\frac{1-\pi_{b}}{\pi_{b}}$, one can find an allocation $C^{0}$ on the $T^{0}$ curve such that $U\left(C^{0}\right)>U(C)$ and $-\vec{\pi}_{a} \cdot\left(C-C^{0}\right)>0$.

\section{- Proof of Proposition 2}

Proof. We only have to verify that no latent intermediary has an incentive to deviate. This clearly implies that there is no profitable deviation for intermediaries offering $(0,0)$ either.

We first remark that whenever one latent intermediary removes his offer, then only the two contracts $\tilde{d}^{0}$ and $\tilde{d}^{L}$ will be available in the economy.

Now, suppose that an intermediary deviates proposing a positive insurance contract. The price of the deviating contract must be strictly smaller than $\frac{1-\pi_{b}}{\pi_{b}}$ for the agent to accept it. It follows that the deviation will only be profitable as long as $e=a$ is selected. Moreover, the price should be greater than $\frac{1-\pi_{a}}{\pi_{a}}$ for the deviator to make profits.

To show that none of these deviations could be profitable, we first argue that the array $\left(C^{0}, L^{0}\right)$ satisfies the following

$$
\left.\left(1-\pi_{a}\right)\left(u^{\prime}\left(c_{1}^{0}\right)-u^{\prime}\left(c_{2}^{0}\right)\right)<\frac{\left(\pi_{a}-\pi_{b}\right)}{\pi_{a}} u^{\prime}\left(\left(1-\pi_{b}\right) c_{1}^{0}\right)+\left(\pi_{b} c_{2}^{0}\right)\right)
$$

which guarantees that $\frac{d U\left(C^{0}\right)}{d U\left(L^{0}\right)}<0$ for small deviations along a line of slope $\frac{-\left(1-\pi_{a}\right)}{\pi_{a}}$. It follows that the slope of $T^{0}$ evaluated in $C^{0}$ is greater than $\frac{1-\pi_{a}}{\pi_{a}} .^{28}$

Let us now take $K=\left(k_{1}, k_{2}\right) \in \mathcal{A}$ to be the agent's optimal consumption allocation at the deviation stage. Clearly, $K$ will be located in the triangle $C^{0}-L^{0}-K^{0}$ in Figure 6 , where $K^{0}$ is on the line of slope $-\frac{1-\pi_{a}}{\pi_{a}}$ passing through $C^{0}$.

$$
\begin{aligned}
& { }^{28} \text { Notice first that because of (10), } \frac{\tau^{L}-\tau^{a}}{\tau^{L^{0}}-\tau^{a}}<\frac{\tau^{L}}{\tau^{L^{0}}} \text { and } \frac{\tau^{C^{0}}-\tau^{a}}{\tau^{C}-\tau^{a}}<\frac{\tau^{C^{0}}}{\tau^{C}} \text {; this implies the following } \\
& \frac{\pi_{b} u^{\prime}\left(l_{2}\right)\left(\tau^{L}-\tau^{a}\right)}{\pi_{b} u^{\prime}\left(l^{0}\right)\left(\tau^{L^{0}}-\tau^{a}\right)} \frac{\pi_{a} u^{\prime}\left(c_{2}^{0}\right)\left(\tau^{C^{0}}-\tau^{a}\right)}{\pi_{a} u^{\prime}\left(c_{2}\right)\left(\tau^{C}-\tau^{a}\right)}<\frac{u^{\prime}\left(l_{2}\right) \tau^{L}}{u^{\prime}\left(l^{0}\right) \tau^{L^{0}}} \frac{u^{\prime}\left(c_{2}^{0}\right) \tau^{C^{0}}}{u^{\prime}\left(c_{2}\right) \tau^{C}}=\frac{u^{\prime}\left(l_{1}\right)}{u^{\prime}\left(l^{0}\right)} \frac{u^{\prime}\left(c_{1}^{0}\right)}{u^{\prime}\left(c_{1}\right)}<1 * 1=1 .
\end{aligned}
$$

Observe that the equality has been derived using the definition of the MRS (in particular $\tau^{L}=\frac{1-\pi_{b}}{\pi_{b}}$ ), while the last inequality coming from the first line of (10). Then, because of (3), one can conclude that $\pi_{a} u^{\prime}\left(c_{2}^{0}\right)\left(\tau^{C^{0}}-\tau^{a}\right)<\pi_{b} u^{\prime}\left(l^{0}\right)\left(\tau^{L^{0}}-\tau^{a}\right)$, which is equivalent to (18). 


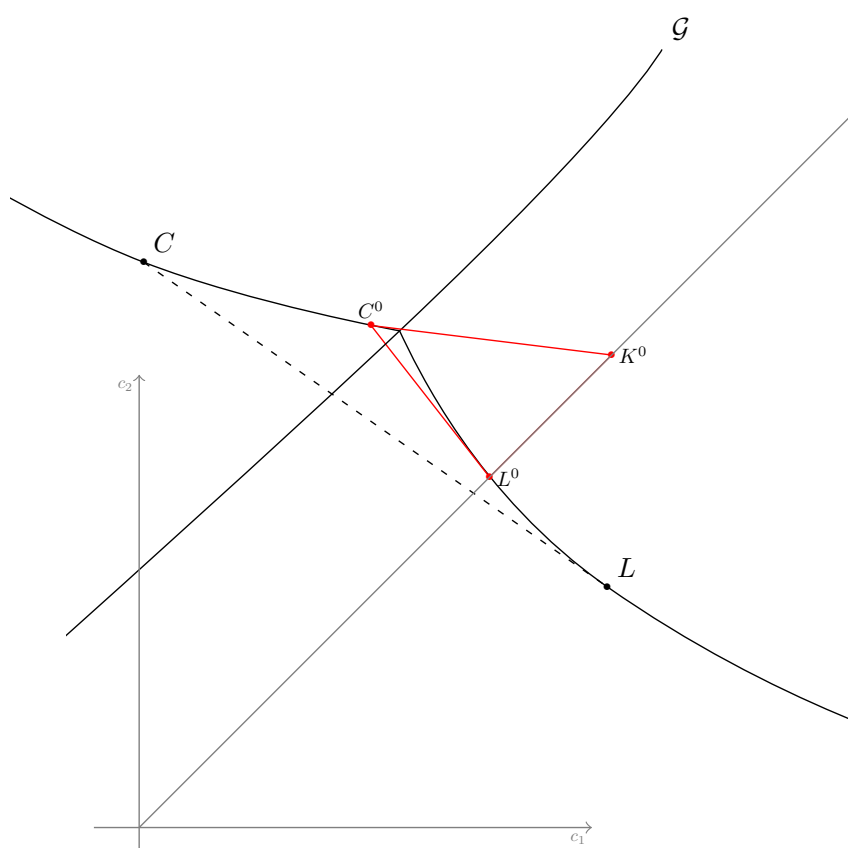

Figure 6: Third best inefficiency

One should observe that if the inequality (18) is verified by any $K \in \mathcal{A}$ in the triangle, then the $T^{0}$ curve will always lie above the $C^{0}-L^{0}-K^{0}$ area. Clearly, this guarantees that there cannot be any incentive for side contracting involving deviations to positive insurance contracts. To show that this indeed the case, we first consider any point $K \in \mathcal{A}$ between $C^{0}$ and $L^{0}$. Then, the utility corresponding to right-hand side of (18) is not affected, while the that associated to the left-hand side is always dominated by $\left(1-\pi_{a}\right)\left(u^{\prime}\left(c_{1}^{0}\right)-u^{\prime}\left(c_{2}^{0}\right)\right)$ making (18) still valid. To extend this result to the other points of the triangle one can use the properties of homothetic preferences. That is, one should observe that the inequality (18) will also be satisfied by any $\alpha K$, with $\alpha \in \mathbb{R}_{+}$.

As a consequence, the curve $\mathcal{T}^{0}$ cannot cross the triangle $C^{0}-L^{0}-\alpha L^{0}$, implying that at $K$ the agent will prefer the feasible allocation $\left(1-\pi_{b}\right) k_{1}+\pi_{b} k_{2}$ to $K$, a contradiction.

In a final step, we remark that if any intermediary deviates through a negative insurance contract, then the only way to make the agent accepting the deviation will be to induce a high effort choice. This indeed makes the deviation not profitable, which constitutes a contradiction.

\section{- Menus and latent contracts}

We show in the following paragraphs how the equilibrium allocation characterized in proposition 1 can as well be supported as a symmetric equilibrium outcome of a simpler game where two competitors (principals) offer menus of alternatives to the agent. We will therefore consider the following game $\Gamma^{M}$. Two principals compete through the menu offers they simultaneously make to a single agent. Given the set $\mathcal{D}^{i}$ of decisions available to the $i-t h$ principal, we let $\mathcal{P}\left(\mathcal{D}^{i}\right)$ be the corresponding set of available menus. ${ }^{29}$ A strategy for the $i-t h$ principal will hence be a map from $\mathcal{P}\left(\mathcal{D}^{i}\right)$ to itself. The extensive form of the game is such that for every array of offered menus, the agent will choose one item from the menu of each competitor and take her (binary) effort decision.

Now, take any equilibrium allocation array $(C, L)$ as described in proposition 1 . Let us consider a situation where each competitor $i=1,2$ offers the same menu of insurance contracts:

\footnotetext{
${ }^{29}$ That is, we take $\mathcal{P}\left(\mathcal{D}^{i}\right)$ to be the Power Set of $\mathcal{D}^{i}$.
} 


$$
\mathcal{M}^{i}=\left\{\mu^{i} \frac{d^{1}}{2}+\lambda^{i} \tilde{d} / \mu^{i} \in[0,1], \lambda^{i} \in[0,1]\right\}, \quad \text { for } i \in\{1,2\}
$$

where $\mu^{i}$ and $\lambda^{i}$ define the consumer's selected item. Clearly, the consumer can achieve the allocation $C$ by selecting $\mu^{1}=\mu^{2}=1$ and $\lambda^{1}=\lambda^{2}=0$. In the menu game, the set of consumption allocations available to the agent when any of the principals removes his offer is represented by the dark area in Figure 7 (left).

In addition, the contract $\tilde{d}=\left(\tilde{d}_{1}, \tilde{d}_{2}\right)$ is selected to satisfy our original condition (7) together with the following:

$$
w_{1}+\frac{d_{1}^{1}}{2}+\tilde{d}_{1}=w_{2}+\frac{d_{2}^{1}}{2}+\tilde{d}_{2}
$$

and

$$
\frac{\left(1-\pi_{b}\right) u^{\prime}\left(q+\Pi \frac{\tau^{b}+1}{\tau^{b}-\tau^{L}}\right)}{\pi_{b} u^{\prime}\left(q-\Pi \frac{\tau^{L}\left(\tau^{b}+1\right)}{\tau^{b}-\tau^{L}}\right)}<\tau^{L}
$$

with $q=w_{1}+\frac{d_{1}^{1}}{2}+\tilde{d}_{1}$ and $\Pi=-\vec{\pi}_{a} \cdot \frac{d^{1}}{2}=-\left(1-\pi_{a}\right) \frac{d_{1}^{1}}{2}-\pi_{a} \frac{d_{2}^{1}}{2} \cdot{ }^{30}$ In this construction, equations (19) and (20) play the same role of (5) and (6) in the original framework. ${ }^{31}$ The first equation implies that if principal 2 removes his offer, the agent can at most get full insurance, and then achieve the allocation $Q=(q, q)$. The equilibrium menus is hence constructed letting each principal offer the additional amount of (latent) insurance $\tilde{d}$, which corresponds to that available in the original game when the active intermediary was withdrawing his offer. The inequality in (20) implies that the allocation $R$ connected to $Q$ by a line of slope $\tau^{L}$ such that $-\vec{\pi}_{a} \cdot(R-Q)=$ $-\vec{\pi}_{a} \cdot \frac{d^{1}}{2}$ falls below the line connecting the origin to $L$ (Figure 7 (right)). ${ }^{32}$
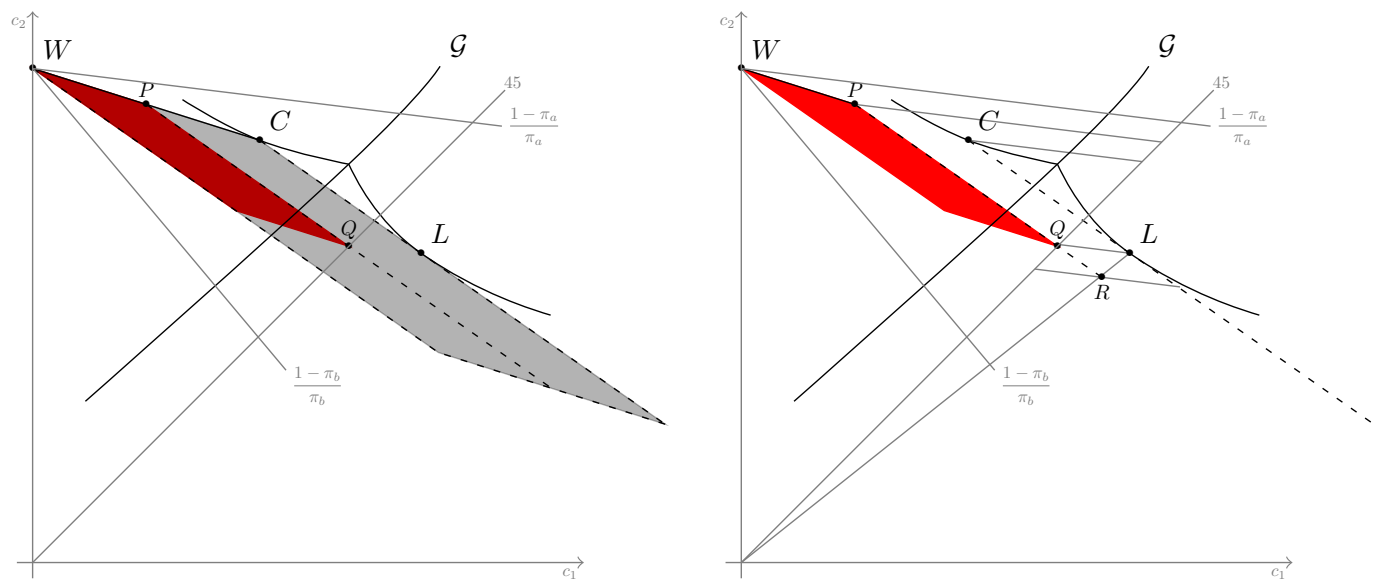

Figure 7: Consumption allocations available in the menu game

To prove that it is an equilibrium for each of the principals to offer the menu $\mathcal{M}^{i}$ and for the agent to select

${ }^{30}$ With the parameters of lemma 1 , condition (20) is verified: $q \approx 37.1637, \Pi \approx \frac{3.85201}{2} \approx 1.92601$ and the computed MRS $\approx 0.859659$ is smaller than 1.

${ }^{31} \mathrm{~A}$ further analysis of the system (1) $-(7)$ together with (19) and (20) shows that $-\vec{\pi}_{a} \cdot \tilde{d}>-\vec{\pi}_{a} \cdot(L-C)=-\vec{\pi}_{a} \cdot d_{2}$.

${ }^{32}$ One can easily check that $R=\left(q+\Pi \frac{\tau^{b}+1}{\tau^{b}-\tau^{L}}, q-\Pi \frac{\tau^{L}\left(\tau^{b}+1\right)}{\tau^{b}-\tau^{L}}\right)$. 
$\mu^{1}=\mu^{2}=1$ and $\lambda^{1}=\lambda^{2}=0$ and to take $e=a$, it is enough to prove that none of the principals can profitably deviate proposing a take-it-or-leave-it offer.

We first argue that there cannot be any profitable deviation inducing $e=a$. Consider principal 1 . When he withdraws his menu offer, the agent can always get to a point like $Q$, on the 45 degree line (equation (19)). Clearly, there is no negative insurance contract which is at a time profitable and such to guarantee the agent a utility greater than $U(Q)$. Consider then a deviation to a positive insurance contract: the price of the deviating contract must be greater than $\frac{1-\pi_{b}}{\pi_{b}}$, for the deviation to be profitable. The agent will hence never have an incentive to trade such a contract and select $e=b$, because the corresponding utility will be always dominated by $U(Q)$.

We now prove that profitable deviations inducing $e=a$ are not possible either.

We first remark that there cannot be profitable deviations through negative insurance contracts. The price of the corresponding contract has to be smaller than $\tau^{a}$, which implies that none of these deviations will be accepted by the agent.

Finally, we have to consider deviations to a positive insurance contract guaranteeing a profit strictly greater than $-\vec{\pi}_{a} \cdot \frac{d^{1}}{2}$. We take $K$ to be the corresponding optimal consumption choice for the agent. For the deviation to be profitable, it should be $K \in \mathcal{A}$, and $U(K) \geq U(Q)$. We denote $p \leq \tau^{L}$ the price of such a deviating contract. One should first notice that $p \leq p^{1}$. If this was not the case, then the $\frac{d^{1}}{2}$ remaining contract would be entirely bought at the deviation stage, and then $K$ would lie below the iso-profit line of slope $-\frac{1-\pi_{a}}{\pi_{a}}$ passing through $C$. Then, conditions (19) and (20) guarantee that there will be enough available insurance for the consumer to achieve her preferred allocation in the $\mathcal{B}$ set. ${ }^{33}$ One could then argue, along the lines of lemma 2 , that there will always be an incentive for the agent to choose $e=b$ at the deviation stage, and this makes the deviation not profitable.

If we consider a deviation such that $p \leq p^{1}$, and any $K \in \mathcal{A}$ verifying $U(K) \geq U(Q)$, then point 3 of lemma 2 can be directly applied to show that the agent will prefer to take low effort, and punish the deviator.

If any of the principals deviates offering a (positive insurance) contract which price is greater than $p^{1}=-\frac{d_{1}}{d_{2}}$, then conditions (19) and (20) guarantee, that there will be enough available insurance for the consumer to achieve her preferred allocation in the $\mathcal{B}$ set. One can therefore conclude, following again lemma 2 , that there will always be an incentive for the agent to choose $e=b$ at the deviation stage, which makes the deviation non profitable.

\section{References}

ArnotT, R., And J. Stiglitz (1993): "Equilibrium in Competitive Insurance Markets with Moral Hazard," mimeo, Boston College.

Attar, A., E. Campioni, A. Chassagnon, and U. Rajan (2008): "Bertrand-Edgeworth competition in non-exclusive markets," mimeo, IDEI Toulouse.

Attar, A., E. Campioni, And G. Piaser (2006): "Multiple lending and constrained efficiency in the credit market," Contributions to Theoretical Economics, 6(1).

Attar, A., D. Majumdar, G. Piaser, And N. Porteiro (2008): “Common agency games: Indifference and separable preferences," Mathematical Social Sciences, 56(1), 75-95.

Attar, A., G. Piaser, And N. Porteiro (2007): “A note on Common Agency models of moral hazard," Economics Letters, 95(2), 278-284.

\footnotetext{
${ }^{33}$ One should also observe that there will always be some available consumption below the line of slope $-\frac{1-\pi_{a}}{\pi_{a}}$ passing through $R$. That is, there will be allocations at the right of $R$ lying below the line connecting the origin to $L$, which guarantees that the corresponding $\gamma L$ allocation is available.
} 
Bisin, A., And D. Guaitoli (2004): "Moral hazard with nonexclusive contracts," Rand Journal of Economics, 2, 306-328.

Bizer, D., And P. DeMarzo (1992): “Sequential Banking,” Journal of Political Economy, 100(1), $41-61$.

HELLWIG, M. (1983): "On moral hazard and non-price equilibria in competitive insurance markets," mimeo, Boston College.

Kahn, C. M., And D. MookherJee (1998): "Competition and Incentives with Nonexclusive Contracts," RAND Journal of Economics, 29(3), 443-465.

Martimort, D., And L. A. Stole (2002): "The revelation and delegation principles in common agency games," Econometrica, 70(4), 1659-1673.

(2003): "Contractual externalities and common agency equilibria," Advances in Theoretical Economics, 3(1).

Parlour, C. A., and U. Rajan (2001): "Competition in Loan Contracts," American Economic Review, 91(5), 1311-1328.

Pauly, M. (1974): "Overinsurance and Public Provision of Insurance: The Roles of Moral Hazard and Adverse Selection," Quarterly Journal of Economics, 88, 44-62.

Simon, L. K., And M. B. STINChOMbe (1995): "Equilibrium Refinement for Infinite Normal-Form Games," Econometrica, 63(6), 1421-1443. 